\title{
Characterization of Ancestral Origin of Cystic Fibrosis of Patients with New Reported Mutations in CFTR
}

\author{
César Paz-y-Miño ${ }^{(D)}{ }^{1}$ Ana Karina Zambrano $\left(D^{1},{ }^{1}\right.$ Juan Carlos Ruiz-Cabezas, ${ }^{2,3,4}$ \\ Isaac Armendáriz-Castillo $\mathbb{D}^{\mathbb{D}}{ }^{1}$ Jennyfer M. García-Cárdenas $\mathbb{D}^{\mathrm{D}}{ }^{1}{ }^{1}$ Santiago Guerrero, ${ }^{1}$ \\ Andrés López-Cortés $\mathbb{I D}^{1}{ }^{1}$ Andy Pérez-Villa $\mathbb{D}$, ${ }^{1}$ Patricia Guevara-Ramírez $\mathbb{D}^{1}{ }^{1}$ \\ Verónica Yumiceba ${ }^{(D)}{ }^{1}$ and Paola E. Leone ${ }^{1}{ }^{1}$ \\ ${ }^{1}$ Centro de Investigación Genética y Genómica, Facultad de Ciencias de la Salud Eugenio Espejo, Universidad UTE, Av. Mariscal \\ Sucre and Mariana de Jesús, Block I, Quito 170129, Ecuador \\ ${ }^{2}$ Universidad de Especialidades Espíritu Santo (UEES), Guayaquil, Ecuador \\ ${ }^{3}$ Instituto de Biomedicina, Universidad Católica de Santiago de Guayaquil, Guayaquil, Ecuador \\ ${ }^{4}$ Instituto Oncológico Nacional de la Sociedad de Lucha Contra el Cáncer (ION-SOLCA), Guayaquil, Ecuador
}

Correspondence should be addressed to César Paz-y-Miño; cesar.pazymino@ute.edu.ec

César Paz-y-Miño and Ana Karina Zambrano contributed equally to this work.

Received 8 July 2019; Accepted 6 May 2020; Published 2 June 2020

Academic Editor: Hugh D. Smyth

Copyright (c) 2020 César Paz-y-Miño et al. This is an open access article distributed under the Creative Commons Attribution License, which permits unrestricted use, distribution, and reproduction in any medium, provided the original work is properly cited.

\begin{abstract}
The incidence of cystic fibrosis (CF) and the frequency of the variants reported for CFTR depend on the population; furthermore, CF symptomatology is characterized by obstructive lung disease and pancreatic insufficiency among other symptoms, which are reliant on the individual's genotype. The Ecuadorian population is a mixture of Native Americans, Europeans, and Africans. That population admixture could be the reason for the new mutations reported in a previous study by Ruiz et al. (2019). A panel of 46 Ancestry Informative Markers was used to estimate the ancestral proportions of each available sample (12 samples in total). As a result, the Native American ancestry proportion was the most prevalent in almost all individuals, except for three patients from Guayaquil with the mutation [c.757G>A:p.Gly253Arg; c.1352G>T:p.Gly451Val] who had the highest European composition.
\end{abstract}

\section{Introduction}

Cystic fibrosis (CF) is an autosomal recessive disorder that has been extensively studied among populations [1]. It is characterized by obstructive lung disease, pancreatic insufficiency, diabetes, and liver disease, among others [2]. The most frequent worldwide mutation in Cystic Fibrosis Transmembrane Conductance Regulator (CFTR) protein gene is c.1521_1523delCTT (p.Phe508del) [3] which originated between 11,000 and 34,000 years ago in Europeans, then it spread across all Europe [4]. CF occurs in 1 out of 2,500 live births with high prevalence in the European ancestry, and the frequency of the heterozygotes has been reported as 1 in 25 in Europeans [5] [6]. There are plenty of studies in $\mathrm{CF}$, yet the majority in Europeans, underrepresenting the
Latin Americans [4] [6] [7] [8]. In the United States, a study reported the CF incidence to be 1 in 9,200 Hispanics and 1 in 10,900 Native Americans, yet the USA has a different population structure to South America [1] [6] [9]. In general, in Latin America, the incidence is 1 per 6,000 live newborns; specifically, Ecuador exhibits an incidence of 1 in 11,252 newborns [10-12].

The Ecuadorian population, located in the northwest of South America, is a mixed population conformed by Native Americans, Europeans who arrived in the $16^{\text {th }}$ century during the conquest, and Africans who came with them as slaves. According to the last census, the population projection for 2020 was estimated as 17,510,643 Ecuadorians. Moreover, Ecuadorian self-identified as "mestizos" 71.9\%, "montubios" 7.4\%, Afro-Ecuadorian 7.2\%, "indígenas" 7\%, "blancos" 
$6.1 \%$, and others $(0.4 \%)$ [13]. There are also reports of the Ecuadorian ancestry using AIMs in the mestizo population where Native American was the most prevalent ancestry (59.6\%), followed by European (28.8\%) and lastly African (11.6\%) [14] [15].

Like other South American studies, Ecuador is underrepresented in cystic fibrosis research, and none of them involve the comparison of the mutations with the population's origin. Paz-y-Miño et.al (1999) reported 10 cases of Ecuadorian CF patients; at least $60 \%$ of the mutations differ from c.1521_ 1523delCTT (p.Phe508del) [16]. Valle et al. (2007) analyzed 62 Ecuadorian CF patients; the most prevalent mutation was F508del (37.1\%) [12]. The last report by Ortiz et al. (2017), which included 48 Ecuadorian individuals with CF, reported F508del with the highest frequency (20.27\%) [17]. These studies, however, are mainly focused on the particular F508 mutation, revealing that the percentage is not relatively high as in Europeans. The incidence and the frequency of the CF mutation depend on the population under study; Ecuadorians are a mestizo population, and the population's composition is not clear yet.

Here, we provide the ancestry origin data of 46 Ancestry Informative Markers of the individuals with the new mutations reported in a previous study of CF patients from Ecuador [18]. We aimed to elucidate if the mutations reported are mainly from European ancestry, due to the previous data of the main incidence.

\section{Main Text}

\subsection{Methods}

2.1.1. Samples and DNA Extraction. Twelve CF patients from Guayaquil (coast) and Cuenca (highland) who were available and had new CFTR disease-causing variants reported in a previous study were selected: one patient from Guayaquil with c.1473T>A:p.Cys491*, one patient from Guayaquil and two from Cuenca with c.2672del:p.Asp891Alafs*15, one patient from Cuenca with c.1486T>C:p.Trp496Arg, and six patients from Guayaquil and one from Cuenca with [c.757G>A:p.Gly253Arg; c.1352G>T:p.Gly451Val] were selected [18]. DNA was extracted using Chelex 100 (Bio-Rad) (10\%) from peripheral blood samples collected on FTA cards (GE Healthcare Life Sciences) and quantified using NanoDrop (Thermo Scientific). To protect the identity of the individuals, the samples were anonymized.

2.1.2. DNA Amplification. PCR amplification of the twelve CF samples and controls (positive: 2800 and negative) was performed using 46 AIMs-INDELs: MID-1470, MID-777, MID-196, MID-881, MID-3122, MID-548, MID-659, MID2011, MID-2929, MID-593, MID-798, MID-1193, MID1871, MID-17, MID-2538, MID-1644, MID-3854, MID2275, MID-94, MID-3072, MID-772, MID-2313, MID-397, MID-1636, MID-51, MID-2431, MID-2264, MID-2256, MID-128, MID-15, MID-2241, MID-419, MID-943, MID159, MID-2005, MID-250, MID-1802, MID-1607, MID1734, MID-406, MID-1386, MID-1726, MID-3626, MID360, MID-1603, and MID-2719 [19], in one multiplex reac- tion and following the standardized protocol of the laboratory. The fragment separation was carried out in 3500 Genetic Analyzers (Applied Biosystems). Data were collected with Data Collection v3 and visualized with GeneMapper v5.

2.2. Statistical Analyses. Data were analyzed with Structure v2.3.4 in order to estimate the ancestral proportions in the population; the runs consisted of a burn-in length of 10,000 followed by 10,000 Markov Chain Monte Carlo (MCMC) interactions. The option used was the admixture model ("use population information to test for migrants"). The cluster considered for the analysis was one to three $(k=1, k=2$, and $k=3$ ) due to the historical background of the Ecuadorian population and according to the cluster identification by Zambrano et al. and Evanno et al. [20] [14].

Principal component analysis (PCA) was built with RStudio v1.1.453 to visualize the CF individuals' structure: the correlation between individuals under analysis and the reference population from HGDP-CEPH (Native Americans, Europeans, and Africans) subset H952 [19].

\section{Results}

The DNA quantification was optimal to perform the PCR (5$20 \mathrm{ng} / \mu \mathrm{l})$. After the amplification, complete profiles were obtained. A total of 339 individuals (reference population and samples) were analyzed, assuming a clustering of three using the information to test for migrants, permuting 10,000 burn-in periods and 10,000 interactions, and a bar plot was obtained showing the main ancestral population analyzed (Figure 1).

Principal component analysis (PCA) results showed the three reference populations clearly differentiate between them. The CF Ecuadorian population is in the middle of them but mainly between the European and Native American reference populations. The two main principal components represented $38.86 \%$ of the total (Figure 2).

A percentage of the ancestral composition of each individual was obtained; as a result, a heterogeneous percentage was found depending on the individual and the region under study, thus clearly showing the admixture of the Ecuadorian population according to history (Table 1). The global ancestry composition of CF patients was the Native American 50\% (standard deviation of 14.03), the European 35\% (standard deviation of 15.5), and the African $11.5 \%$ (standard deviation of 7.82). The Native American ancestry was the first origin of almost all individuals, except for three patients from Guayaquil with the mutation [c.757G>A:p.Gly253Arg; c.1352G>T:p.Gly451 Val] with the highest European composition.

\section{Discussion}

The present study is the first report of the ancestral composition of CF Ecuadorian patients with new CFTR mutations. There are plenty of CF studies among different populations that revealed the differences between gender, age, and symptoms in CF patients. Some studies compared CF patients of different ages and gender describing that the incidence 


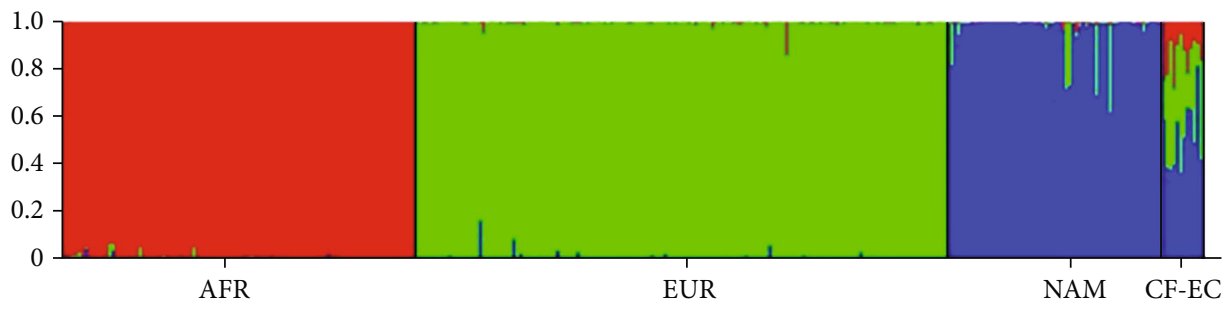

FIGURE 1: . Bar plot grouped by population identification. AFR: African ancestry; EUR: European ancestry; NAM: Native American ancestry; CF-EC: Ecuadorian cystic fibrosis patients. Three inferred clusters $(K=3)$.

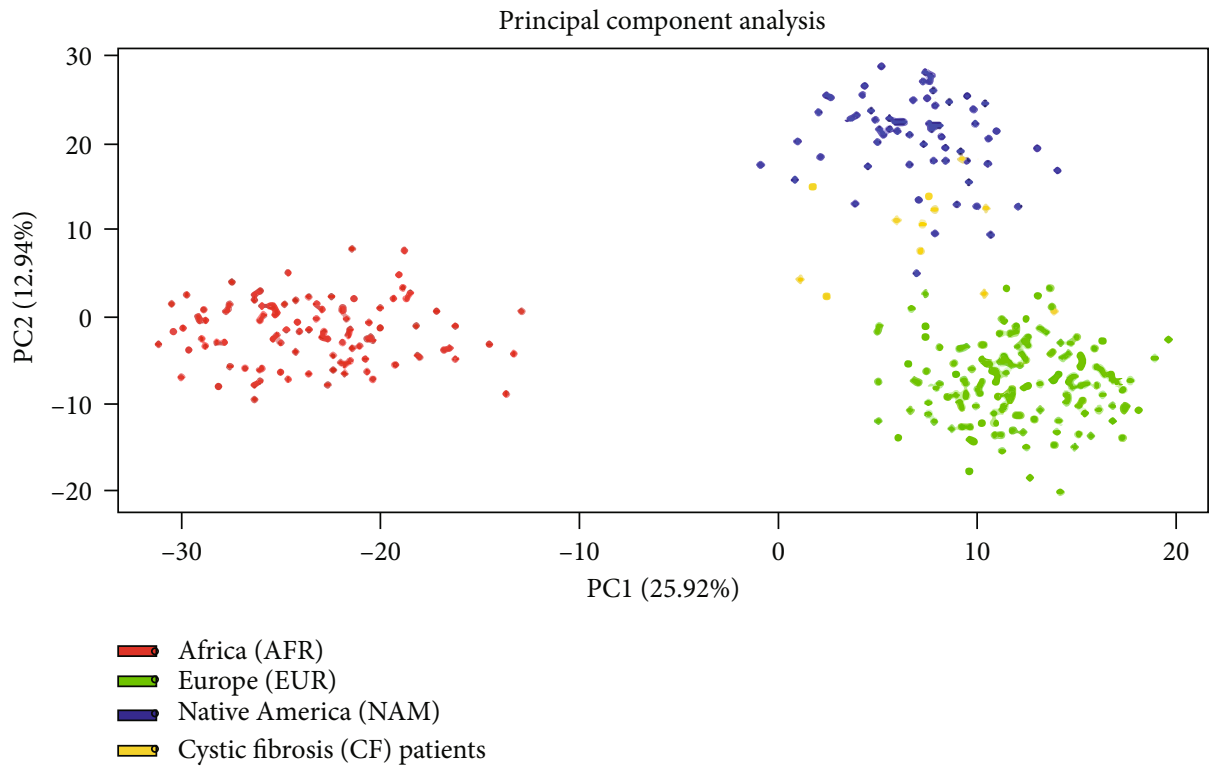

Figure 2: . Principal component analysis of Ecuadorian cystic fibrosis patients.

TABle 1: Percentage of the ancestral composition of each individual under study.

\begin{tabular}{|c|c|c|c|c|c|}
\hline Patient & AFR & EUR & NAM & Mutation & Mutations' reference \\
\hline 01 & 24.6 & 16.7 & 58.7 & c.1473T>A:p.Cys491* & [18] \\
\hline 02 & 7.6 & 43.5 & 48.9 & c.2672del:p.Asp891Alafs $* 15$ & [18] \\
\hline 03 & 21.6 & 14.8 & 63.6 & c.2672del:p.Asp891Alafs $* 15$ & {$[18]$} \\
\hline 04 & 16 & 42.2 & 41.8 & c.2672del:p.Asp891Alafs $* 15$ & [18] \\
\hline 05 & 9.1 & 8.9 & 82 & c.1486T>C:p.Trp496Arg & [18] \\
\hline 06 & 22.5 & 39.6 & 37.9 & [c.757G>A:p.Gly253Arg; c.1352G>T:p.Gly451Val] & [18] \\
\hline 07 & 7.2 & 55.3 & 37.5 & [c.757G>A:p.Gly253Arg; c.1352G>T:p.Gly451 Val] & [18] \\
\hline 08 & 28.2 & 31.9 & 39.9 & [c.757G>A:p.Gly253Arg; c.1352G>T:p.Gly451 Val] & [18] \\
\hline 09 & 5 & 58.7 & 36.3 & [c.757G>A:p.Gly253Arg; c.1352G>T:p.Gly451 Val] & [18] \\
\hline 10 & 11.9 & 37.2 & 50.9 & [c.757G>A:p.Gly253Arg; c.1352G>T:p.Gly451 Val ] & [18] \\
\hline 11 & 9.3 & 32.1 & 58.6 & [c.757G>A:p.Gly253Arg; c.1352G>T:p.Gly451 Val] & [18] \\
\hline 12 & 11 & 25.7 & 63.3 & [c.757G>A:p.Gly253Arg; c.1352G>T:p.Gly451 Val] & [18] \\
\hline
\end{tabular}

AFR: African ancestry; EUR: European ancestry; NAM: Native American ancestry.

of $\mathrm{CF}$ in Europe is higher in children than in adults, approximately $4 \mathrm{CF}$ children per 3 adults and around 1.1 males per each female [21] [22].

There are reports that evidenced CF prevalence differs depending on ethnicity. For instance, the incidence in Native
Americans, whites and black individuals is $37.2,38.8$, and 17.1 per 100,000 , respectively [23]. Moreover, other studies revealed the incidence among diverse ethnicities: as an example, the prevalence of CF reported by Rohlfs et al. (2011) is 1 in 242 Asian, 1 in 28 Caucasian, 1 in 59 Hispanic, and 1 in 70 
Native American [2]. That study clearly revealed the ethnic differences in the incidence and the distribution of $\mathrm{CF}$ worldwide.

In Ecuador, there are studies about the ancestral origin; for instance, the Ecuadorian was reported to be composed of 59.6\% Native American, 28.8\% European, and lastly $11.6 \%$ African origin [14] [15].

In addition to the variable predisposition of $\mathrm{CF}$ among populations, reports exhibit a total of 2,063 mutations listed on the CFTR mutation database [24], while in the CFTR2 database, the most recent file updated on 8 December 2017 shows a total of 374 variants [25]. Those variants were identified in different populations in diverse frequencies. For instance, the frequencies of the most common variant c.1521_1523delCTT (p.Phe508del) depend on ethnicity; it was reported as $72 \%$ in US Caucasians, $~ 41 \%$ in African Americans, and $18 \%$ in Iranians, yet it also differs among Caucasians [26] [2] [27] [1] [28].

There are some mutations that have been commonly reported in ethnic groups; as examples, c.1624G > T (p.Gly542X) was reported in $43 \%$ of Turkish origin [29], while in a study in Peruvian patients, the frequency was $6.9 \%$ [30], c.3846G $>\mathrm{A}$ (p.Trp1282Ter) was reported in $43 \%$ of Ashkenazi patients [31], c. $2988+1 G>A(3120+1 G>A)$ was reported in $12.3 \%$ of native African $\mathrm{CF}$ patients [32] [33], and c.3909C>G (p.Asn1303Lys) was described in $1.7 \%$ of the total number of CF analyzed from Europeans and the United States population [34], while in the Algerian population, the frequency was 20\% [35]; c.1652G>A (p.Gly551Asp) presented a frequency of $3 \%$ in a north Brazilian population [36]. Furthermore, some mutations have been found in a specific ethnic group, like c.16C $>\mathrm{G}$ (p.Leu6Val) was found in one Argentinian and c.3294G $>C$ (p.Trp1098Cys) was found in one Mexican, among other variations described [4]; c.3276C $>$ G (p.Tyr1092Ter) was found in Jews from Iraq [31] [2].

In conclusion, the identification of ethnicity-dependent mutations would be an important aspect of CF testing in Ecuador. The present study exhibited a greater ancestral composition of Native American, followed by European and lastly African; the mixed population origin could possibly explain the new CF mutations reported.

4.1. Limitations. Although we have found the ancestral proportions of the majority of CF patients with new mutations previously reported, we could not access all the samples due to the available conditions of the patients. Moreover, a larger CF patient study with the commonly reported mutation should be conducted to better approximate the ancestral proportions of the patients.

\section{Abbreviations}

CF: $\quad$ Cystic fibrosis

CFTR: Cystic Fibrosis Transmembrane Conductance Regulator

AIMs: Ancestry Informative Markers

INDELs: Insertions and deletions

PCA: Principal component analysis
AFR: African ancestry

EUR: European ancestry

NAM: Native American ancestry.

\section{Data Availability}

The authors confirm that the data supporting the findings of this study are available within the article. The complete raw data that support the findings of this study are available from the corresponding author upon reasonable request.

\section{Ethical Approval}

The patients were registered with the Ecuadorian Cystic Fibrosis Foundation at Guayaquil and Cuenca. The investigation was approved with the number 2018-127E by the "Comité de Ética de Investigación en Seres Humanos Universidad San Francisco de Quito."

\section{Consent}

Patients provided informed consent, including the paternal signed authorization to participate in the study.

\section{Conflicts of Interest}

The authors declare that they have no competing interests.

\section{Authors' Contributions}

César Paz-y-Miño was responsible for coordination and followed up with the development of the article. Ana Karina Zambrano was responsible for the design, experimental procedure, data analysis, and writing. Juan Carlos RuizCabezas participated in the samples collection and written edition. Isaac Armendáriz-Castillo participated in the written edition. Jennyfer García-Cárdenas participated in the written edition. Santiago Guerrero participated in the written edition. Andrés López-Cortés participated in the written edition. Andy Pérez-Villa participated in the writing and formatting of the article. Patricia Guevara-Ramírez participated in the written edition. Verónica Yumiceba participated in the written edition. Paola E. Leone participated in the written edition. César Paz-y-Miño and Ana Karina Zambrano contributed equally to this work.

\section{Acknowledgments}

The authors are grateful to the patients for their selfless participation in the present study and to all the people and institutions involved in the development of the present study.

\section{References}

[1] R. A. Heim, E. A. Sugarman, and B. A. Allitto, "Improved detection of cystic fibrosis mutations in the heterogeneous U.S. population using an expanded, pan-ethnic mutation panel," Genetics in Medicine, vol. 3, no. 3, pp. 168-176, 2001. 
[2] E. M. Rohlfs, Z. Zhou, R. A. Heim et al., "Cystic fibrosis carrier testing in an ethnically diverse US population," Clinical Chemistry, vol. 57, no. 6, pp. 841-848, 2011.

[3] S. Raskin, L. Pereira-Ferrari, F. C. Reis et al., "Incidence of cystic fibrosis in five different states of Brazil as determined by screening of p.F508del, mutation at the CFTR gene in newborns and patients," Journal of Cystic Fibrosis, vol. 7, no. 1, pp. 15-22, 2008, https://www.cysticfibrosisjournal.com/arti cle/S1569-1993(07)00036-7/pdf.

[4] M. M. Pérez, M. C. Luna, O. H. Pivetta, and G. Keyeux, "CFTR gene analysis in Latin American CF patients: heterogeneous origin and distribution of mutations across the continent," Journal of Cystic Fibrosis, vol. 6, no. 3, pp. 194-208, 2007.

[5] L. Pereira, S. Raskin, A. A. Freund et al., "Cystic fibrosis mutations R1162X and 2183AA $<$ FONT FACE $=$ Symbol ${ }^{\circledR}</-$ FONT $>\mathrm{G}$ in two southern Brasilian states," Genetics and Molecular Biology, vol. 22, no. 3, pp. 291-294, 1999.

[6] World Health Organization, "The molecular genetic epidemiology of cystic fibrosis," pp. 1-24, 1983, https://www.who.int/ genomics/publications/en/HGN_WB_04.02_report.pdf.

[7] L. Ioannou, B. J. McClaren, J. Massie et al., "Population-based carrier screening for cystic fibrosis: a systematic review of 23 years of research," Genetics in Medicine, vol. 16, no. 3, pp. 207-216, 2014.

[8] S. Ogino, P. Flodman, R. B. Wilson, B. Gold, and W. W. Grody, "Risk calculations for cystic fibrosis in neonatal screening by immunoreactive trypsinogen and CFTR mutation tests," Genetics in Medicine, vol. 7, no. 5, pp. 317-327, 2005.

[9] A. Hamosh, S. C. FitzSimmons, M. Macek, M. R. Knowles, B. J. Rosenstein, and G. R. Cutting, "Comparison of the clinical manifestations of cystic fibrosis in black and white patients," The Journal of Pediatrics, vol. 132, no. 2, pp. 255-259, 1998.

[10] P. Hernández and F. González-Andrade, "Guía de práctica clínica (GPC) y manual de procedimientos fibrosis quística," p. 94, 2013, Jun 2018 http://instituciones.msp.gob.ec/docu mentos/Guias/fibrosis.pdf.

[11] L. V. R. F. Silva Filho, C. Castaños, and H. H. Ruíz, "Cystic fibrosis in Latin America-improving the awareness," Journal of Cystic Fibrosis, vol. 15, no. 6, pp. 791-793, 2016.

[12] E. P. Valle, R. I. Burgos, J. R. Valle, D. Egas Béjar, and J. C. Ruiz-Cabezas, "Analysis of CFTR gene mutations and cystic fibrosis incidence in the Ecuadorian population," Investigación Clínica, vol. 48, no. 1, pp. 91-98, 2007.

[13] INEC Poster INEC, "Censo de población y vivienda," 2010, http://www.ecuadorencifras.gob.ec/censo-de-poblacion-yvivienda.

[14] A. K. Zambrano, A. Gaviria, S. Cobos-Navarrete et al., "The three-hybrid genetic composition of an Ecuadorian population using AIMs- InDels compared with autosomes, mitochondrial DNA and Y chromosome data," Scientific Reports, vol. 9, no. 1, p. 9247, 2019, http://www.nature.com/scientifi creports.

[15] A. K. Zambrano, A. Gaviria, M. Vela et al., "Ancestry characterization of Ecuador's highland mestizo population using autosomal AIM-INDELs," Forensic Science International: Genetics Supplement Series, vol. 6, pp. e477-e478, 2017.

[16] C. Paz-y-Mino, J. C. Perez, R. Burgos, M. V. Davalos, and P. E. Leone, "The $\Delta$ F508 mutation in Ecuador, South America," Human Mutation, vol. 14, no. 4, pp. 348-350, 1999.

[17] S. C. Ortiz, S. J. Aguirre, S. Flores, C. Maldonado, J. Mejía, and L. Salinas, "Spectrum of CFTR gene mutations in Ecuadorian cystic fibrosis patients: the second report of the p.H609R mutation," Molecular Genetics \& Genomic Medicine, vol. 5, no. 6, pp. 751-757, 2017.

[18] J. C. Ruiz-Cabezas, F. Barros, B. Sobrino et al., "Mutational analysis of CFTR in the Ecuadorian population using nextgeneration sequencing," Gene, vol. 696, pp. 28-32, 2019.

[19] R. Pereira, C. Phillips, N. Pinto et al., "Straightforward inference of ancestry and admixture proportions through ancestry-informative insertion deletion multiplexing," PLoS One, vol. 7, no. 1, p. e29684, 2012.

[20] G. Evanno, S. Regnaut, and J. Goudet, "Detecting the number of clusters of individuals using the software STRUCTURE: a simulation study," Molecular Ecology, vol. 14, no. 8, pp. 2611-2620, 2005.

[21] P. A. di Sant'Agnese and P. B. Davis, "Cystic fibrosis in adults: 75 cases and a review of 232 cases in the literature," The American Journal of Medicine, vol. 66, no. 1, pp. 121-132, 1979.

[22] J. McCormick, M. W. Green, G. Mehta, F. Culross, and A. Mehta, "Demographics of the UK cystic fibrosis population: implications for neonatal screening," European Journal of Human Genetics, vol. 10, no. 10, pp. 583-590, 2002, May 2018, https://www.nature.com/articles/5200850.pdf.

[23] K. A. Spoonhower and P. B. Davis, "Epidemiology of cystic fibrosis," Clinics in Chest Medicine, vol. 37, no. 1, pp. 1-8, 2016.

[24] R. Dorfman, "Cystic Fibrosis Mutation Database: Statistics," Cystic Fibrosis Mutation Database, 2015, Apr 2019, http:// www.genet.sickkids.on.ca/StatisticsPage.html.

[25] The Hospital for Sick Children, Johns Hopkins University, The Hospital for Sick Children, "CFTR2 clinical and functional translation if CFTR,” 2019, Apr 2019, https://www. cftr2.org/.

[26] G. E. Palomaki, S. C. Fitzsimmons, and J. E. Haddow, "Clinical sensitivity of prenatal screening for cystic fibrosis via _CFTR carrier testing in a United States panethnic population," Genetics in Medicine, vol. 6, no. 5, pp. 405-414, 2004.

[27] E. A. Sugarman, E. M. Rohlfs, L. M. Silverman, and B. A. Allitto, "CFTR mutation distribution among U.S. Hispanic and African American individuals: Evaluation in cystic fibrosis patient and carrier screening populations," Genetics in Medicine, vol. 6, no. 5, pp. 392-399, 2004.

[28] R. Alibakhshi, R. Kianishirazi, J.-J. Cassiman, M. Zamani, and H. Cuppens, "Analysis of the CFTR gene in Iranian cystic fibrosis patients: identification of eight novel mutations," Journal of Cystic Fibrosis, vol. 7, no. 2, pp. 102-109, 2008.

[29] I. Lerer, M. Sagi, G. R. Cutting, and D. Abeliovich, "Cystic fibrosis mutations delta F508 and G542X in Jewish patients," Journal of Medical Genetics, vol. 29, no. 2, pp. 131-133, 1992.

[30] R. Aquino, A. Protzel, J. Rivera et al., "Frequency of the most common mutations of the CFTR gene in Peruvian patients with cystic fibrosis using the ARMS-PCR technique," Revista Peruana de Medicina Experimental y Salud Pública, vol. 34, no. 1, pp. 62-69, 2017.

[31] A. Quint, I. Lerer, M. Sagi, and D. Abeliovich, "Mutation spectrum in Jewish cystic fibrosis patients in israel: implication to carrier screening," American Journal of Medical Genetics Part A, vol. 136A, no. 3, pp. 246-248, 2005.

[32] C. Padoa, A. Goldman, T. Jenkins, and M. Ramsay, "Cystic fibrosis carrier frequencies in populations of African origin," Journal of Medical Genetics, vol. 36, no. 1, pp. 41-44, 1999, May 2018, http://www.ncbi.nlm.nih.gov/pubmed/9950364. 
[33] M. Macek Jr., A. Mackova, A. Hamosh et al., "Identification of common cystic fibrosis mutations in African-Americans with cystic fibrosis increases the detection rate to 75\%," American Journal of Human Genetics, vol. 60, no. 5, pp. 1122-1127, 1997.

[34] L. Osborne, G. Santis, M. Schwarz et al., "Incidence and expression of the N1303K mutation of the cystic fibrosis (CFTR) gene," Human Genetics, vol. 89, no. 6, pp. 653-658, 2004.

[35] O. Loumi, M. Baghriche, M. Delpech, J. C. Kaplan, and T. Bienvenu, "Analysis of the complete coding region of the CFTR gene in ten Algerian cystic fibrosis families," Human Heredity, vol. 49, no. 2, pp. 81-84, 1999.

[36] F. G. de Araújo, F. C. Novaes, N. P. C. dos Santos et al., "Prevalence of deltaF508, G551D, G542X, and R553X mutations among cystic fibrosis patients in the north of Brazil," Brazilian Journal of Medical and Biological Research, vol. 38, no. 1, pp. 11-15, 2005. 